\title{
$\mathrm{Cd}_{1-x} \mathrm{Fe}_{x} \mathrm{Te}$ TERNARY CRYSTAL FORMATION STUDIED BY RESONANT PHOTOEMISSION
}

\author{
E. Guziewicz, B.J. Kowalski, Z. Golacki, B.A. Orlowski \\ Institute of Physics, Polish Academy of Sciences \\ Al. Lotników 32/46, 02-668 Warszawa, Poland
}

R.L. JOHNSON

Universität Hamburg, II Institut für Experimentalphysik

Luruper Chaussee 149, 22761 Hamburg, Germany

\section{AND J. MASEK}

Institute of Physics, Czech Academy of Sciences

18040 Praha 8, Czech Republic

\begin{abstract}
A resonant photoemission was used to study the ternary crystal formation, when small amount of $\mathrm{Fe}$ atoms was deposited (in one monolayer range of thickness) on the clean $\mathrm{CdTe}(100)$ surface. The constant initial state spectra taken near the $\mathrm{Fe} 3 p-3 d$ transition after $\mathrm{Fe}$ deposition and then again after heating process show the existence of two Fano-like resonance. The differences of the energy distribution curves taken for both resonance and antiresonance, respectively, allow us to distinguish two kind of $\mathrm{Fe} 3 d$ contributions to the valence band: one derived from the metallic $\mathrm{Fe}$ islands on the surface and the second - derived from the Fe atoms built into the $\mathrm{Cd}_{1-x} \mathrm{Fe}_{x} \mathrm{Te}$ crystal.
\end{abstract}

PACS numbers: 79.60.-i, 68.35.Fx

In the very first stages of the metal atoms deposition on clean semiconductor surface the Shottky barrier can be formed if the deposited atoms create the islands of metal and then monolayer of it. If the deposited atoms react with the substrate the doping of semiconductor surface region appears and the ternary crystal layer can be created on the surface. The paper presents results of a unique experimental method very sensitive on surface electronic properties to distinguish between these two possible positions in a case of Fe atoms deposited on CdTe clean surface. Recently many of investigations are sacrificed to study the process of formation of semimagnetic semiconductors (SMSC) in the region near the surface, when transition metal (TM) atoms were deposited on the clean II-VI or III-V semiconductor's surface [1-3]. 
The experimental method of study of TM $3 d$ contribution into the valence band density of states (DOS) is a resonant photoemission spectroscopy in which TM $3 d$ electrons excited selectively and locally according to the equation

$$
\mathrm{TM} 3 p^{6} 3 d^{N} \rightarrow \mathrm{TM} 3 p^{5} 3 d^{N+1} \rightarrow \mathrm{TM} 3 p^{6} 3 d^{N-1}+\text { e. }
$$

This additional contribution to the measured spectrum of photoemitted electrons appears only for resonant energy $h \nu$ corresponding to $\mathrm{Fe} 3 p-3 d$ transition. The effect is described as a Fano-type resonance [4]. The differences in energy distribution curves (EDC) taken for resonant and antiresonant energy show the contribution of TM $3 d$ electrons to the SMSC valence band density of states.

The experiment was carried out in a very clean ultra high vacuum conditions using the synchrotron radiation obtained from the beam line Flipper II of the Storage Ring DORIS II in HASYLAB in Hamburg, Germany. The synchrotron radiation was used in the photon energy range of $50-130 \mathrm{eV}$.

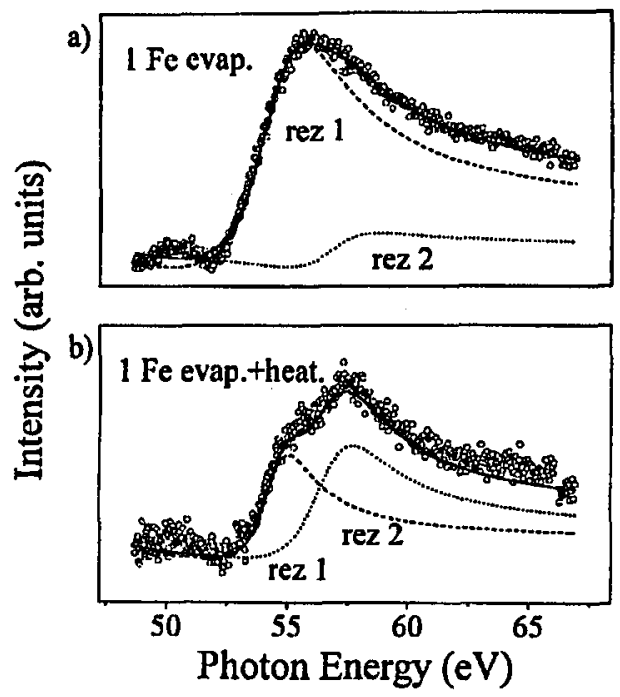

Fig. 1. Constant initial states spectra of $\mathrm{CdTe}(100)$ crystal measured for initial state $4.6 \mathrm{eV}$ below the Fermi level: (a) after evaporation of $\mathrm{Fe}$, (b) after heating of obtained $\mathrm{CdTe}(100) / \mathrm{Fe}$ crystal.

The experiment consisted of two technological processes: (i) $\mathrm{Fe}$ atoms in the monolayer range were deposited on the clean $\mathrm{CdTe}(100)$ surface; (ii) after the $\mathrm{Fe}$ deposition the heating of the sample led to the diffusion of $\mathrm{Fe}$ atoms into the CdTe crystal and the layer of the semimagnetic semiconductor $\mathrm{Cd}_{1-x} \mathrm{Fe}_{x} \mathrm{Te}$ was created in the region near the surface. In order to investigate the energy of Fe $3 p-3 d$ resonance the constant initial state (CIS) spectrum was taken after evaporation and heating processes. Figure 1 presents the CIS curves and result of the deconvolution of the spectra on two Fano-like curves. The CIS curve measured after the Fe evaporation process (Fig. 1a) shows the existence of mainly one Fano 
resonance with the maximum at an energy $55.5 \mathrm{eV}$. After heating the shape of CIS curve changes (Fig. 1b), one can observe that the CIS curve consists of two contributions: apart from that with the maximum at an energy $55.5 \mathrm{eV}$ appears the second one with the maximum at an energy $57.2 \mathrm{eV}$. Therefore we believe that the resonance at $55.5 \mathrm{eV}$ is derived from the $\mathrm{Fe}$ atoms adsorbed on the crystal surface ( $\mathrm{Fe}$ islands) whereas the resonance at $57.2 \mathrm{eV}$ is derived from the Fe atoms built into the $\mathrm{Cd}_{1-x} \mathrm{Fe}_{x} \mathrm{Te}$ crystal. Our conclusion is consistent with the results obtained by Lad and Henrich [5], where it was found out that the Fano resonance energy is lower for metallic $\mathrm{Fe}$ than for $\mathrm{Fe}^{2+}$ and $\mathrm{Fe}^{3+}$ ions.
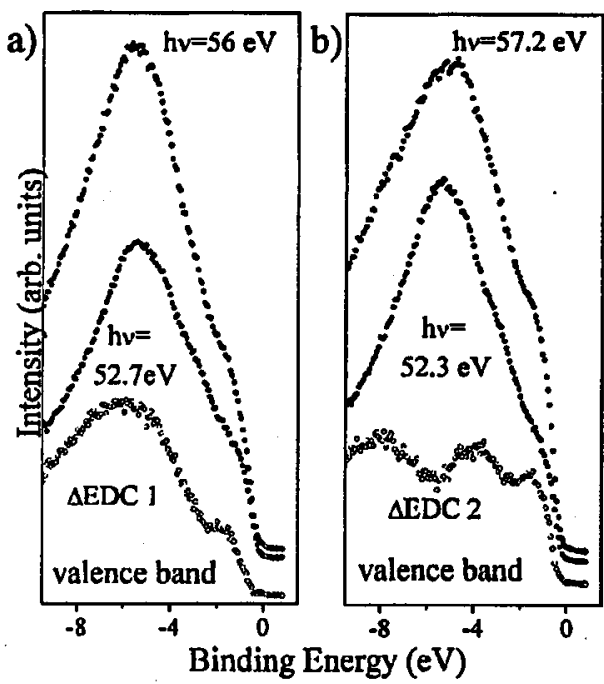

Fig. 2. Energy distribution curve of $\mathrm{CdTe}(100) / \mathrm{Fe}$ crystal (solid circles) taken on resonance and antiresonance energy of both resonances $(56 \mathrm{eV}$ and $57.2 \mathrm{eV})$. The difference spectrum $(\triangle \mathrm{EDC})$ is a measure of the $\mathrm{Fe} 3 d$ partial density of states (open circles): (a) resonance $\operatorname{EDC}(h \nu=56 \mathrm{eV})$, antiresonance $\operatorname{EDC}(h \nu=52.7 \mathrm{eV})$ and $\triangle \mathrm{EDC}$ of the first resonance ( $\mathrm{Fe}$ evaporated on the surface), (b) resonance EDC $(h \nu=57.2 \mathrm{eV})$, antiresonance $\mathrm{EDC}(h \nu=52.3 \mathrm{eV})$ and $\triangle \mathrm{EDC}$ of the second resonance (Fe from $\mathrm{Cd}_{1-x} \mathrm{Fe}_{x} \mathrm{Te}$ layer).

Figures $2 \mathrm{a}$ and $\mathrm{b}$ present the photoemission measurements taken after heating of $\mathrm{CdTe} / \mathrm{Fe}$ crystal. In Fig. 2a the EDC curves taken near resonant $(56 \mathrm{eV})$ and antiresonant $(52.7 \mathrm{eV})$ energies of the first resonance are shown. The result of the subtraction of the resonant and antiresonant EDCs $(\triangle \mathrm{EDC})$ is plotted below. We expect that $\triangle E D C$ in Fig. 2a ( $\triangle$ EDC 1 ) represents Fe $3 d$-derived partial valence band spectrum of iron atoms absorbed on the surface. In the curve $\triangle$ EDC 1 two structures appear: the narrow one at $1.7 \mathrm{eV}$ and the wide at $6 \mathrm{eV}$ below the Fermi level. The former is a structure typical of $\mathrm{Fe} 3 d$ states, whereas the latter is a satellite usually observed in transition metals like $\mathrm{Fe}, \mathrm{Co}, \mathrm{Ni}[6]$. A resonant and antiresonant EDC of the second resonance $(57.2 \mathrm{eV})$ are presented in Fig. $2 \mathrm{~b}$. 
The curve $\triangle E D C 2$ shows the Fe $3 d$-derived partial valence band spectrum of $\mathrm{Cd}_{1-x} \mathrm{Fe}_{x} \mathrm{Te}$ created in the region near the crystal surface. It reveals three contributions with maximum located at: $7.9,4.0$ and $1.75 \mathrm{eV}$ below the Fermi level. The positions of the maxima are consistent with those obtained from the preliminary theoretical calculations obtained by means of tight binding method, which predicts that $\mathrm{Fe} 3 d$-states in $\mathrm{Cd}_{1-x} \mathrm{Fe}_{x} \mathrm{Te}$ crystal should give a triple contribution to the valence band density of states: near the edge of the valence band (narrow structure derived from $e_{\mathrm{g} \downarrow}$ states), a wide structure with maximum about $2.0 \mathrm{eV}$ below the first one (derived from $t_{2 \mathrm{~g} \uparrow}$ states) and the third one, which is situated between 4.2 and $6.2 \mathrm{eV}$ below the valence band edge (derived from $t_{2 \mathrm{~g} \uparrow}$ and $e_{\mathrm{g} \downarrow}$ states). The results are also consistent with these obtained for $\mathrm{Cd}_{1-x} \mathrm{Fe}_{x} \mathrm{Se}$ bulk crystals $[7,8]$.

In the first stages of Fe atoms deposition on CdTe (100) surface the two states of $\mathrm{Fe}$ atoms were distinguished. One is ascribed to the metallic $\mathrm{Fe}$ islands and the other to the $\mathrm{Fe}$ atoms built into $\mathrm{Cd}_{1-x} \mathrm{Fe}_{x} \mathrm{Te}$ alloy.

The work was supported partially by the Committee for Scientific Research grant PB No. 2P03B08910.

\section{References}

[1] A. Mycielski, J. Appl. Phys. 63, 3279 (1988).

[2] B.A. Orlowski, J.P. Lacharme, N. Safta, C.A. Sebenne, in: Proc. 4th Int. Conf. Formation of Semic. Interfaces, Jülich (Germany) 1993, Eds. B. Lengerer, H. Luth, W. Monch, J. Pollmann, World Scientific, Singapore 1993, p. 235.

[3] A. Wall, A. Raisanen, G. Haugstad, L. Vanzetti, A. Franciosi, Phys. Rev. B 44, 8185 (1991).

[4] U. Fano, Phys. Rev. 124, 1866 (1961).

[5] R.J. Lad, V.E. Henrich, Phys. Rev. B 39, 13478 (1989).

[6] C. Guillot, Y. Ballu, J. Paigne, J. Lecante, K.P. Jain, P. Thiry, R. Pinchaux, Y. Petroff, L.M. Falicov, Phys. Rev. Lett. 39, 1632 (1977).

[7] M. Taniguchi, Y. Ueda, I. Morisada, Y. Murashita, T. Ohta, I. Souma, Y. Oka, Phys. Rev. B 41, 3069 (1990).

[8] R. Denecke, L. Ley, J. Fraxedas, Phys. Rev. B 47, 13197 (1993). 